\title{
Assessing the lifetime performance Index using exponentiated Frechet distribution with the progressive first-failure-censoring scheme
}

\author{
Ahmed Abo-Elmagd Soliman, Essam Al-Sayed Ahmed, Ahmed Hamed Abd Ellah, \\ Al-Wageh Ahmed Farghal
}

Mathematics Department, Faculty of Science, Sohag University, Sohag 82524, Egypt

\section{Email address:}

a_a_sol@hotmail.com (A. Abo-Elmagd Soliman), ahmhamed@hotmail.com (A. H. A. Ellah), esam_alsyd@yahoo.com (E.Al-Sayed Ahmed), alwageh_ahmed@yahoo.com (Al-Wageh A. F.)

\section{To cite this article:}

Ahmed Abo-Elmagd Soliman, Essam Al-Sayed Ahmed, Ahmed Hamed Abd Ellah, Al-Wageh Ahmed Farghal. Assessing the Lifetime Performance Index Using Exponentiated Frechet Distribution with the Progressive First-Failure-Censoring Scheme. American Journal of Theoretical and Applied Statistics. Vol. 3, No. 6, 2014, pp. 167-176. doi: 10.11648/j.ajtas.20140306.11

\begin{abstract}
Process capability analysis has been widely used to monitor the performance of industrial processes. In practice, lifetime performance index $\mathrm{C}_{\mathrm{L}}$ is a popular means to assess the performance and potential of their processes, where $\mathrm{L}$ is the lower specification limit. This study constructs the maximum likelihood (ML) and the Bayesian estimators of $\mathrm{C}_{\mathrm{L}}$ for the exponentiated Frechet (EF) model with progressive first-failure-censoring scheme. These estimates are then used for constructing a confidence interval for $\mathrm{C}_{\mathrm{L}}$. The MLE and the Bayesian estimators of $\mathrm{C}_{\mathrm{L}}$ are then utilized to develop a new hypothesis testing procedure in the condition of known L. Finally, we give a practical example and the Monte Carlo simulation study to illustrate the use of the testing procedure under given significance level.
\end{abstract}

Keywords: Exponentiated Frechet Distribution, Progressive First-Failure Censored Samples, Lifetime Performance Index, Hypothesis Testing, Maximum Likelihood, Bayes Estimates

\section{Introduction}

Statistical distributions are very useful in identifying and predicting real world phenomena. One of them is the extreme value distribution. It has been extensively used to model natural phenomena, modeling lifetimes and also in material strengths. The Frechet distribution is one of three kinds of general extreme value distribution, known as the extreme value distribution of type II. It was first developed and applied to the flood flows by Frechet [1]. It is widely used in many disciplines such as earthquake engineering, floods, rain fall, queues in supermarkets, sea currents, wind speeds and track race records, see Kotz and Nadarajah [2].

In recent years, several standard life time distributions have been generalized via exponentiation. Examples of such exponentiated distributions are the exponentiated Weibull family, the exponentiated exponential, the exponentiated Rayleigh, the exponentiated Gumbel and the exponentiated Pareto family of distributions. Nadarajah and Kotz [3] introduced a new lifetime model named the Exponentiated
Frechet distribution (EF). It is a generalization of the standard Frechet distribution. The EF distribution is referred to in the literature as the inverse of exponentiated Weibull distribution. In this article, we focus on a two-parameter exponentiated Frechet (EF) distribution. For a full discussion and some of its mathematical properties, see Nadarajah and Kotz [3]. The probability density function (PDF), cumulative distribution function (CDF) of a random variable $X$ having two parameter EF distribution are respectively given by

$$
f(x)=\theta \alpha x^{-(\alpha+1)} e^{-x^{-\alpha}}\left(1-e^{-x^{-\alpha}}\right)^{\theta-1}, x>0,
$$

and

$$
F(x)=1-\left(1-e^{-x^{-\alpha}}\right)^{\theta}, x>0, \alpha, \theta>0,
$$

where $\alpha$ and $\theta$ are shape parameters. In this paper, a distribution will be denoted by $\mathrm{EF}(\alpha, \theta)$.

Before a new product is launched to the market, life tests are 
often required to assess its reliability. In life testing experiments, the experimenter may not always be in a position to observe the life times of all the products or items put on test. This may be because of time limitation or other restrictions such as money, material resources, mechanical or experimental difficulties on data collection. Therefore, the need for censoring has thus arisen and the censored samples are thus obtained. There exist various types of censored samples including Type-II and progressive Type-II, progressive first-failure censored samples. Among the different censoring schemes, the progressive Type-II censoring scheme has most widely been used particularly in reliability analysis and survival analysis. It is preferred to traditional Type-II censoring scheme. Progressive censoring is useful in both industrial life testing applications and clinical settings; it allows the removal of surviving experimental units before the termination of the test. It is seen by many experimenters as an efficient way of reducing costs due to total time on test, or due to failing units. It is also used widely due to its flexibility in experimental design, and the fact that it contains the usual order statistics and Type-II right censoring as special cases. There has been a considerable discussion on progressive censoring and its applications in the literature; see Balakrishnan and Aggarwala [4] and Balakrishnan [5].

In some cases, the lifetime of products is quite long and so the experimental time of the Type-II censoring scheme can still be too long. In order to give an efficient experiment, the other test methods are proposed by statisticians where one of them is the first-failure censoring scheme, introduced in Johnson [6]. In this censoring scheme, $m \times n$ items are divided to $m$ equal groups. Then, the life test is conducted by testing each of these groups simultaneously and terminated when the first failure is observed in each groups. The first-failure-censored sampling plan has an advantage in terms of shorter test time and a saving of resources. But, it does not allow for sets to be removed from the test at the points other than the final termination point. Hence $\mathrm{Wu}$ and Kus [7] combined the first-failure censored scheme and progressively type II right censored scheme in order to propose a new life test plan called the progressive first-failure censoring scheme which is more efficient in lifetime studies. Therefore, the new scheme has an advantage in terms of shorter test time, a saving of resources, and in which a specific fraction of individuals at risk may be removed from the experiment at each of several ordered failure times. The progressive first-failure-censored sampling plan is illustrated as follows:

Suppose that $m$ is the number of failures observed before termination and $n$ independent groups with $k$ items within each group are put in a life test. $R_{1}$ groups and the group in which the first failure is observed are randomly removed from the test as soon as the first failure say $X_{1}$ has occurred, $R_{2}$ groups and the group in which the second failure is observed are randomly removed from the test as soon as the first failure say $X_{2}$ has occurred, and finally $R_{m}(m \leq n)$ groups and the group in which the $m$ th failure is observed are randomly removed from the test as soon as the $m$ th failure say $X_{m}$ has occurred. Then
$X_{1}<X_{2}<\ldots<X_{m}$ are called the progressive first-failure-censored order statistics with censoring scheme $R=\left(R_{1}, R_{2}, \ldots, R_{m}\right)$, where $n=m+\sum_{i=1}^{m} R_{i}$. The familiar complete, type II right censored, first-failure-censored, and progressively type II right censored samples are special cases of the progressive first-failure-censored sampling plan. If $R_{i}=0, i=1, \ldots, m$, then the progressive first- failurecensored sampling plan reduces to the first-failure-censored sampling plan. If $k=1$, then the progressive first- failure censored sampling plan reduces to the progressively type II right censored sampling plan. If $k=1$ and $R_{i}=0, i=1, \ldots, m \quad(n=m)$ then, the progressive first-failure-censored sampling plan reduces to the complete sampling plan. Also, if $k=1, R_{i}=0, i=1, \ldots, m-1$ and $R_{m}=n-m$ then the progressive first-failure-censored sampling plan reduces to type II right censored sampling plan. For more details see $\mathrm{Wu}$ and Kus [7].

If the failure times of the $n \times k$ items originally in the test are from a continuous population with distribution function $F(x)$ and probability density function $f(x)$, the joint probability density function for $x_{1: m: n: k}^{R}, x_{2: m: n: k}^{R}, \ldots, x_{m: m: n: k}^{R}$ is given by

$$
\begin{aligned}
& f_{1,2, \ldots, m}\left(x_{1: m: n: k}^{R}, x_{2: m: n: k}^{R}, \ldots, x_{m: m: n: k}^{R}\right)= \\
& A k^{m} \prod_{i=1}^{m} f\left(x_{i, m, n}\right)\left[1-F\left(x_{i, m, n}\right)\right]^{k\left(R_{i}+1\right)-1},
\end{aligned}
$$

where

$$
A=n\left(n-1-R_{1}\right)\left(n-2-R 1-R_{2}\right) \ldots\left(n-\sum_{i=1}^{m-1}\left(R_{i}+1\right)\right) .
$$

Statistical inferences on the parameters of failure time distributions under progressive first-failure censoring have been studied by several authors such as Wu and Huang [8] and Soliman et al. [9].

In the manufacturing industry, process capability indices are utilized to assess whether product quality meets the required level. For instance, Montgomery [10] or Kane [11] proposed the process capability index $C_{L}$ for evaluating the lifetime performance of electronic components, where $L$ is the lower specification limit, since the lifetime of electronic components exhibits the larger the better quality characteristic of time orientation. In recent years, there have been many works on the statistical inference for $C_{L}$ based on some different samples from different Statistical distributions. For instance, Tong et al. [12] constructed the uniformly minimum variance unbiased estimator (UMVUE) of $C_{L}$ and proposed a hypothesis testing procedure for the complete sample from a one-parameter exponential distribution. For a type II right censored sample with a two-parameter exponential distribution, Wu et al. [13] provided the maximum-likelihood estimator of $C_{L}$ and utilized this estimator to construct a 
hypothesis testing procedure. Lee et al. [14] also proposed a hypothesis testing procedure based on a MLE of $C_{L}$ to evaluate product quality for exponential distribution under the progressively type II right censored sample. Based on progressively first-failure censored sample coming from the Weibull distribution with a known shape parameter Ahmadi et al. [15] obtained the statistical inference for $C_{L}$ and developed a hypothesis testing procedure. Lee et al. [16] constructed MLE and Bayesian estimator of $C_{L}$ under assuming the conjugate prior distribution and SE loss function, LINEX loss function, and GE loss function based on the upper record values from the Rayleigh distribution. Also, Ahmadi et al. [17] have discussed the Bayesian and non-Bayesian procedures for the estimation of the lifetime performance index based on generalized order statistics from exponential distribution.

In this paper, we assume that the lifetimes of products follow two-parameter EF distribution and develop statistical inference for the unknown lifetime performance index $C_{L}$ based on progressive first-failure-censored. The rest of the paper is organized as follows. In Section 2, some properties of the lifetime performance index $C_{L}$ under the EF distribution and its relationship with the conforming rate $P_{r}$ are presented. In Sections 3, 4 and 5 we consider the problems of estimating and hypotheses testing on the $C_{L}$ based on a progressive first-failure-censored sample via the ML and Bayesian approaches. Two illustrative examples and Monte Carlo simulation study are given in Section 6 to assess the behavior of the lifetime performance index $C_{L}$. Finally, concluding remarks are given in Section 7.

\section{The Lifetime Performance Index and the Conforming Rate}

Suppose that the lifetime $X$ of products has the two-parameter EF distribution with the PDF and CDF, given respectively by (1) and (2). Using the transformation $Y=-\ln \left(1-\exp \left(-X^{-\alpha}\right)\right)$, the distribution of $Y$ is a exponential distribution with the PDF and CDF as

$$
\left.f_{Y}(y, \theta)=\theta \exp (-\theta y)\right), y>0, \theta>0,
$$

and

$$
\left.F_{Y}(y, \theta)=1-\exp (-\theta y)\right), y>0, \theta>0 .
$$

And the failure rate function $r(y)$ is defined by

$$
r(y)=\frac{f_{Y}(y)}{1-F_{Y}(y)}=\theta .
$$

The data transformation $Y=-\ln \left(1-\exp \left(-X^{-\alpha}\right)\right)$, for $\mathrm{X}>0$, is one-to-one and strictly increasing, so data set of $\mathrm{X}$ and transformed data set of $Y$ have the same effect in assessing the lifetime performance of products.

Let $L$ be the lower specification limit for the lifetime of products. Montgomery [10] developed a capability index
$C_{L}$ as follows:

$$
C_{L}=\frac{\mu-L}{\sigma}
$$

where $\mu$ denotes the process mean and $\sigma$ represents the process standard deviation. This lifetime performance index can be used to assess the performance of the lifetime of products. The mean and standard deviation of the new lifetime of products are given by $\mu=E(y)=\frac{1}{\theta}$ and $\sigma^{2}=\operatorname{Var}(y)=\frac{1}{\theta^{2}}$. Hence the capability index $C_{L}$ can be reduced as

$$
C_{L}=\frac{\frac{1}{\theta}-L}{\frac{1}{\theta}}=1-\theta L
$$

we can see that the index $C_{L}>0$ when $\theta<(1 / L)$ and $C_{L}<0$ when $\theta>(1 / L)$. We can also see that the smaller the failure rate $\theta$, the larger is the lifetime performance index $C_{L}$. Therefore, the lifetime performance index $C_{L}$ can accurately assess the lifetime performance of products. If the new lifetime of a product exceeds the lower specification limit (i.e. $Y \geq L$ ), then the product is labelled as a conforming product. Otherwise, the product is labelled as a non-conforming product. The conforming rate can be defined as

$$
\begin{aligned}
P_{r} & =P(Y \geq L)=\int_{L}^{\infty} \theta \exp (-\theta y) d y=\exp (-\theta L) \\
& =\exp \left(C_{L}-1\right), \quad-\infty<C_{L}<1
\end{aligned}
$$

Apparently, there is a strictly increasing relationship existing between conforming rat $P_{r}$ and the lifetime performance index $C_{L}$. The values of $C_{L}$ and the corresponding conforming rates $P_{r}$ are listed in Table 1.

\section{Maximum Likelihood Estimation of $C_{L}$}

Let $\quad \mathrm{x}_{1 \text { :m:n:k}}<\mathrm{x}_{2: \mathrm{m}: \mathrm{n}: \mathrm{k}}<\ldots<\mathrm{x}_{\mathrm{m}: \mathrm{m}: \mathrm{n}: \mathrm{k}}$. be the progressive first-failure censored sample from the two parameter $\mathrm{EF}$ distribution with PDF and $\mathrm{CDF} f(x)$ and $F(x)$, respectively. Substituting from (1) and (2) into (3), the likelihood function is given by

$$
L_{x}(\alpha, \theta \mid x)=C k^{m} \theta^{m} \alpha^{m} \prod_{i=1}^{m} x_{i}^{-\alpha-1} \mathrm{e}^{-X_{i}^{-\alpha}}\left(1-\mathrm{e}^{-x_{i}^{-\alpha}}\right)^{\theta k\left(R_{i}+1\right)-1} .
$$

The log-likelihood function can be obtained from (10) as

$$
\begin{aligned}
\ell_{x}(\alpha, \theta \mid x)= & \ln C+m \ln k+m \ln \theta+m \ln \alpha- \\
& \sum_{i=1}^{m}(\alpha+1) \ln x_{i}-\sum_{i=1}^{m} x_{i}^{-\alpha}+ \\
& \sum_{i=1}^{m} \theta\left(k\left(R_{i}+1\right)-1\right) \ln \left(1-\mathrm{e}^{-x_{i}^{-\alpha}}\right)
\end{aligned}
$$


Consequently, likelihood equations of $\alpha$ and $\theta$ are obtained as

$$
\begin{aligned}
& \frac{\partial \ell_{x}(\alpha, \theta \mid x)}{\partial \alpha}=\frac{m}{\alpha}-\sum_{i=1}^{m}\left(1-x_{i}^{-\alpha}\right) \ln x_{i}- \\
& \sum_{i=1}^{m} \frac{k \theta\left(\left(R_{i}+1\right)-1\right) \mathrm{e}^{-x_{i}^{-\alpha}} X_{i}^{-\alpha} \ln x_{i}}{\left(1-\mathrm{e}^{-x_{i}^{-\alpha}}\right)}=0,
\end{aligned}
$$

and

$$
\frac{\partial \ell_{x}(\alpha, \theta \mid x)}{\partial \theta}=\frac{m}{\theta}+\sum_{i=1}^{m} k\left(R_{i}+1\right) \ln \left(1-\mathrm{e}^{-x_{i}^{-\alpha}}\right)=0 .
$$

It follows, from Equation (13), that

$$
\hat{\theta}_{M L}=-\frac{m}{\sum_{i=1}^{m} k\left(R_{i}+1\right) \ln \left(1-\mathrm{e}^{-x_{i}^{\hat{\alpha}_{M L}}}\right)} .
$$

And $\hat{\alpha}_{M L}$ is the solution of

$$
\begin{aligned}
& \frac{m}{\hat{\alpha}_{M L}}-\sum_{i=1}^{m}\left(1-x_{i}^{-\hat{\alpha}_{M L}}\right) \ln x_{i}-\left(-\frac{m}{\sum_{i=1}^{m} k\left(R_{i}+1\right) \ln \left(1-\mathrm{e}^{-x_{i}^{\hat{\alpha}_{M L}}}\right)}\right) \\
& k \sum_{i=1}^{m} \frac{\left(\left(R_{i}+1\right)-1\right) \mathrm{e}^{-x_{i}^{-\hat{\alpha}_{M L}}} x_{i}^{-\hat{\alpha}_{M L} \ln x_{i}}}{\left(1-\mathrm{e}^{-x_{i}^{-\hat{\alpha}_{M L}}}\right)}=0,
\end{aligned}
$$

We can use iterative techniques such as a NewtonRaphson type algorithm to obtain the estimate $\hat{\alpha}_{M L}$, once we obtain $\hat{\alpha}_{M L}$, the maximum likelihood estimators of $\theta \quad\left(\hat{\theta}_{M L}\right)$ can be obtained from (14). The initial values for the parameters are important but are not hard to obtain by using graphical techniques, see Balakrishnan and Kateri (2008).

By using the transformation $Y=-\ln \left(1-\exp \left(-X^{-\alpha}\right)\right)$, we assume that $\alpha$ is known which can be obtained by using the graphical method or by using ML method.

$Y_{1: m: n: k}<Y_{2: m: n: k}<\ldots<Y_{m: m: n: k}$ will be the corresponding progressive first-failure censored sample from the oneparameter exponential distribution with PDF (4) and censoring scheme $R=\left(R_{1}, R_{2}, \ldots, R_{m}\right)$. Substituting from (4) and (5) into (3), the likelihood function is then given by

$$
L_{y}(\theta \mid y)=C k^{m} \theta^{m} e^{-\theta W}, W=k \sum_{i=1}^{m}\left(R_{i}+1\right) y_{i} .
$$

The log-likelihood function of $\theta$ is obtained from (16) as

$$
\ell_{y}=\ln C+m \ln k+m \ln \theta-\theta W .
$$

To find the value of $\theta$ that maximizes (17), we differentiate (17) with respect to $\theta$ and set the resulting equation to zero. Therefore the MLE of $\theta$ is

$$
\hat{\theta}=\frac{m}{W} .
$$

By the invariance property of the ML estimators, the ML estimator for $C_{L}$ is obtained from Equation (8) as

$$
\hat{C}_{L_{M L}}=1-\frac{m L}{W} \text {. }
$$

It can be notice that $2 \theta \mathrm{W}$ follows the chi-square distribution with $2 m$ degrees of freedom, denoted by $\chi_{(2 m)}^{2}$, see for instance Ahsanullah [18] and Ahmadi et al. [15].

\section{Bayes Estimation of $C_{L}$}

Based on such a progressive first-failure censored sample, in this section we apply the Bayesian approach to compute estimates for $\theta$ and $C_{L}$. In lifetime data analysis, such prior knowledge is usually summarized into a prior density, denoted by $\pi(\cdot)$. For the $\operatorname{Exp}(\theta)$ distribution, a natural conjugate prior density for $\theta$ is the gamma prior density, i.e.

Table 1. The lifetime performance index $C_{L}$ v.s. the conforming rate $P_{r}$.

\begin{tabular}{llllllll}
\hline$C_{L}$ & $P_{r}$ & $C_{L}$ & $P_{r}$ & $C_{L}$ & $P_{r}$ & $C_{L}$ & $P_{r}$ \\
\hline$-\infty$ & 0.00000 & -3.00 & 0.01832 & 0.15 & 0.42741 & 0.60 & 0.67032 \\
-9.00 & 0.00004 & -2.50 & 0.03019 & 0.20 & 0.44933 & 0.65 & 0.70469 \\
-8.00 & 0.00012 & -2.00 & 0.04979 & 0.25 & 0.47237 & 0.70 & 0.74082 \\
-7.00 & 0.00033 & -1.50 & 0.08208 & 0.30 & 0.49659 & 0.75 & 0.77880 \\
-6.00 & 0.00091 & -1.00 & 0.13534 & 0.35 & 0.52205 & 0.80 & 0.81873 \\
-5.00 & 0.00248 & -0.50 & 0.22313 & 0.40 & 0.54881 & 0.85 & 0.86071 \\
-4.50 & 0.00409 & 0.00 & 0.36788 & 0.45 & 0.57695 & 0.90 & 0.90484 \\
-4.00 & 0.00673 & 0.05 & 0.38674 & 0.50 & 0.60653 & 0.95 & 0.95123 \\
-3.50 & 0.01111 & 0.10 & 0.40657 & 0.55 & 0.63763 & 1.00 & 1.0000 \\
\hline
\end{tabular}

$$
\pi(\theta)=\frac{b^{a}}{\Gamma(a)} \theta^{a-1} e^{-b \theta}, \theta>0, a>0, b>0
$$

where the hyperparameters $a$ and $b$ are chosen to reflect prior knowledge about the unknown parameter $\theta$.

An easy way for choosing the $a$ and $b$ is to consider the mean and the variance of the prior distribution of the lifetime performance index $C_{L}$. Let $E\left(C_{L}\right)=\mu_{0}$ and $v\left(C_{L}\right)=\sigma_{0}^{2}$, where $E\left(C_{L}\right)=1-a b L$ and $v\left(C_{L}\right)=a b^{2} L^{2}$. Hence $b=\frac{\sigma_{0}^{2}}{\left(1-\mu_{0}\right) L}$ and $a=\frac{\left(1-\mu_{0}\right)^{2}}{\sigma_{0}^{2}}$

A joint density function of the data $\mathrm{Y}$ and $\theta$ can be obtained by combining the likelihood function in (16) and the prior density (20) as below.

$$
\pi^{*}(\theta, \underline{y})=\frac{b^{a}}{\Gamma(a)} \theta^{a+m-1} e^{-\theta(b+W)} .
$$

Bayesian inference is based on the posterior distribution 
which is simply the ratio of the joint density function to the marginal distribution function. Hence, the posterior density function of $\theta$ given the data is

$$
\pi^{*}(\theta \mid \underline{y})=\frac{(b+W)^{a+m}}{\Gamma(a+m)} \theta^{a+m-1} e^{-\theta(b+W)},
$$

that is, $\theta \mid y \sim$ gamma $(a+m, b+W)$.

Lemma1: It can be notice that If $W^{\prime}=b+k \sum_{i=1}^{m}\left(R_{i}+1\right) y_{i}$ then $2 \theta W^{\prime}$ follows the chi-square distribution with $2(m+a)$ degrees of freedom, denoted by $x_{2(m+a)}^{2}$.

Proof:

Let $Z=2 \theta W^{\prime}$ and by using the change of variables (see [Casella and Berger [19], pp. 184--185]), we obtain the PDF of $Z$ as

$$
\begin{aligned}
& f_{Z}(z)=\boldsymbol{\pi}^{*}\left(\frac{Z}{2 W} \mid y_{1: m: n}, \ldots, y_{m: m: n}\right)\left\|J_{z}\right\| \\
& =\frac{Z^{a+m}}{\Gamma(a+m) 2^{a+m}}\left(\frac{Z}{2 W}\right)^{-1} e^{-\frac{Z}{2}}\left(\frac{1}{2 W}\right) \\
& =\frac{1}{2^{\left(\frac{2(a+m)}{2}\right)} \Gamma\left(\frac{2(a+m)}{2}\right)} Z^{\left(\frac{2(a+m)}{2}\right)-1} e^{-\frac{Z}{2}} \text {. }
\end{aligned}
$$

So we have $Z=2 \theta W^{\prime} \sim \chi_{2(m+a)}^{2}$. The posterior density function of $C_{L}$ given the data is

$$
\begin{aligned}
\pi^{*}\left(C_{L} \mid \underline{y}\right) & =\boldsymbol{\pi}^{*}\left(\left(\frac{1-C_{L}}{L}\right) \mid \underline{y}\right)\left\|J_{C_{L}}\right\| \\
& =\frac{(b+W)^{a+m}}{\Gamma(a+m) L^{a+m}}\left(1-C_{L}\right)^{a+m-1} e^{-\left(1-C_{L}\right)\left(\frac{b+W}{L}\right)} .
\end{aligned}
$$

The Bayes estimator shall be considered under loss function which is also essential in Bayesian estimations. One of loss functions is the squared error loss function (SEL) which is symmetrical in nature. This loss function gives equal weight to estimation errors that are the same regardless of whether the loss obtained has either overestimated or underestimated the parameter or the problem being investigated. It is well known that the Bayes estimator for the parameter under SEL loss function $L\left(\delta, C_{L}\right)=\left(\delta-C_{L}\right)^{2}$ is the mean of the posterior density. Then, under the SEL function, and by using (24) the Bayes estimate of $C_{L}$, is given by

$$
\begin{aligned}
\hat{C}_{L B} & =\int_{-\infty}^{1} C_{L} \pi^{*}\left(C_{L} \mid \underline{y}\right) d C_{L} \\
& =\frac{(b+W)^{a+m}}{\Gamma(a+m) L^{a+m}} \int_{-\infty}^{1} C_{L}\left(1-C_{L}\right)^{a+m-1} e^{-\left(1-C_{L}\right)\left(\frac{b+W}{L}\right)} d C_{L}, \\
& =\left[1-\frac{(a+m) L}{b+W}\right] .
\end{aligned}
$$

\section{Hvpotheses Testing Procedure for the}

$$
C_{L}
$$

In this section, we construct a statistical testing procedure to assess whether the lifetime performance index reaches the required level. Let the required level be $c$. The process is capable if the lifetime performance index is larger than $c$. Then, the statistical hypothesis is set up as follows: $H_{0}: C_{L} \leq c$ (the process is not capable) vs. $H_{1}: C_{L}>c$ (the process is capable). Testing the above hypothesis with significance level $\gamma$ is equivalently finding the $(1-\gamma) 100 \%$ lower confidence bound for $C_{L}$.

\subsection{Testing Procedure for the $C_{L}$ Based on $M L E$}

By using $\hat{C}_{L_{M L}}$, the MLE of $C_{L}$ as the test statistic, given the specified significance level $\gamma$, the level $(1-\gamma)$ one-sided confidence interval for $C_{L}$ can be derived as follows.

By using the pivotal quantity $2 \theta W \sim \chi_{(2 m)}^{2}$, and $c_{1}=\operatorname{CHIINV}(1-\gamma, 2 m)$ which represents the lower $1-\gamma$ percentile of $\chi_{(2 m)}^{2}$, we obtain

$$
\begin{aligned}
& P\left(2 \theta W \leq c_{1} \mid y_{1: m: n}, \ldots, y_{m: m: n}\right)=1-\gamma \\
& \Rightarrow P\left(C_{L} \geq 1-L\left(\frac{c_{1}}{2 W}\right) \mid y_{1: m: n}, \ldots, y_{m: m: n}\right)=1-\gamma \\
& \Rightarrow P\left(C_{L} \geq 1-\left(1-\hat{C}_{L_{M L}}\right)\left(\frac{c_{1}}{2 m}\right) \mid y_{1: m: n}, \ldots, y_{m: m: n}\right)=1-\gamma .
\end{aligned}
$$

Hence, the level $100(1-\gamma) \%$ lower confidence bound for $C_{L}$ can be derived

$$
L B_{M L}=1-\left(1-\hat{C}_{L_{M L}}\right)\left(\frac{c_{1}}{2 m}\right) .
$$

If the performance index value, $c \notin\left[L B_{M L}, \infty\right)$ it is concluded that the lifetime performance index of the product meets the required level.

\subsection{Testing Procedure for the $C_{L}$ Based on Bayes Estimate}

By using $\hat{C}_{L B}$, the Bayes estimate of $C_{L}$ as the test statistic, the level $(1-\gamma)$ one-sided confidence interval for $C_{L}$ can be derived as follows. From Lemma 1 and Eq. (23), we have

$$
\begin{aligned}
& P\left(2 \theta W^{\prime} \leq c_{2} \mid y_{1: m: n}, \ldots, y_{m: m: n}\right)=1-\gamma . \\
& \Rightarrow P\left(\theta \leq\left(\frac{c_{2}}{2 W^{\prime}}\right) \mid y_{1: m: n}, \ldots, y_{m: m: n}\right)=1-\gamma \\
& \Rightarrow P\left(C_{L} \geq 1-L\left(\frac{c_{2}}{2 W^{\prime}}\right) \mid y_{1: m: n}, \ldots, y_{m: m: n}\right)=1-\gamma
\end{aligned}
$$


Then,

$$
P\left(C_{L} \geq 1-\left(1-\hat{C}_{L B}\right)\left(\frac{c_{2}}{2(a+m)}\right)\right)=1-\gamma .
$$

Where $c_{2}=\mathrm{CHIINV}(1-\gamma, 2(m+a))$ is the function which represents the lower $100(1-\gamma)$ the percentile of $x_{2(m+a)}^{2}$.

Therefore, the level $100(1-\gamma) \%$ lower credible bound for $C_{L}$ can be derived

$$
L B_{B}=1-\left(1-\hat{C}_{L B}\right)\left(\frac{c_{2}}{2(a+m)}\right) .
$$

In the Bayesian approach, the managers can then employ the confidence interval to determine whether the business performance adheres to the required level. The proposed testing procedure about $C_{L}$ can be organized as follows:

Step 1: Let the data transformation $Y_{i}=-\ln \left(1-\exp \left(-X_{i}^{-\alpha}\right)\right)$,

$\alpha>0, \quad i=1,2, \ldots, m$ for progressive first- failure censored sample.

Step 2: Determine the lower lifetime limit $L$ for products and performance index value $c$, then the testing null hypothesis $H_{0}: C_{L} \leq c$ and the alternative hypothesis

$$
H_{1}: C_{L}>c \text { is constructed. }
$$

Step 3: Specify a significance level $\gamma$.

Step 4: Calculate the level $100(1-\gamma) \%$ one- sided credible interval $\left[L B_{B}, \infty\right)$ for $C_{L}$, using $(30) \quad L B_{B}=1-\left(1-\hat{C}_{L B}\right)\left(\frac{c_{2}}{2(a+m)}\right)$.

Step 5: The decision rule of statistical test is provided as

follows: If the performance index value $c \notin\left[L B_{B}, \infty\right)$, it is concluded that the lifetime performance index of the Product meets the required level.

\section{Numerical Computations}

Modern statistical problems require a mix of theoretical and application techniques for implementing and understanding statistical inferential methods. Previous sections dealt with the analytical technique and this section focuses on the numerical one through real and simulated examples. Also; we perform extensive Monte Carlo simulations to compare the performance of the different estimators, mainly with respect to their mean-squared errors (MSEs) for different sample sizes and for different parameter values.

\subsection{Illustrative Examples}

Wingo data (Real data): In this example, a complete sample from a clinical trial describe a relief time (in hours) for 50 arthritic patients given by Wingo [20] and used recently by Wu et al. [21] is selected. The data are given in Table 2. We have computed the Kolmogorov-Smirnov (KS) distance between the empirical and the fitted distribution functions. It is 0.11 and the associated $p$-value is 0.53 . Since the $p$-value is quite high, we cannot reject the null hypothesis that the data is coming from the EF distribution. Also, we plot both the empirical survival function (ESF) and the estimated survival functions in Fig. 1 and we found that the EF fits the data very well.

Table 2. Relief time (in hours) for 50 arthritic patients.

\begin{tabular}{llllllllll}
\hline 0.70 & 0.84 & 0.58 & 0.50 & 0.55 & 0.82 & 0.59 & 0.71 & 0.72 & 0.61 \\
0.62 & 0.49 & 0.54 & 0.36 & 0.36 & 0.71 & 0.35 & 0.64 & 0.84 & 0.55 \\
0.59 & 0.29 & 0.75 & 0.46 & 0.46 & 0.60 & 0.60 & 0.36 & 0.52 & 0.68 \\
0.80 & 0.55 & 0.84 & 0.34 & 0.34 & 0.70 & 0.49 & 0.56 & 0.71 & 0.61 \\
0.57 & 0.73 & 0.75 & 0.44 & 0.44 & 0.81 & 0.80 & 0.87 & 0.29 & 0.50 \\
\hline
\end{tabular}

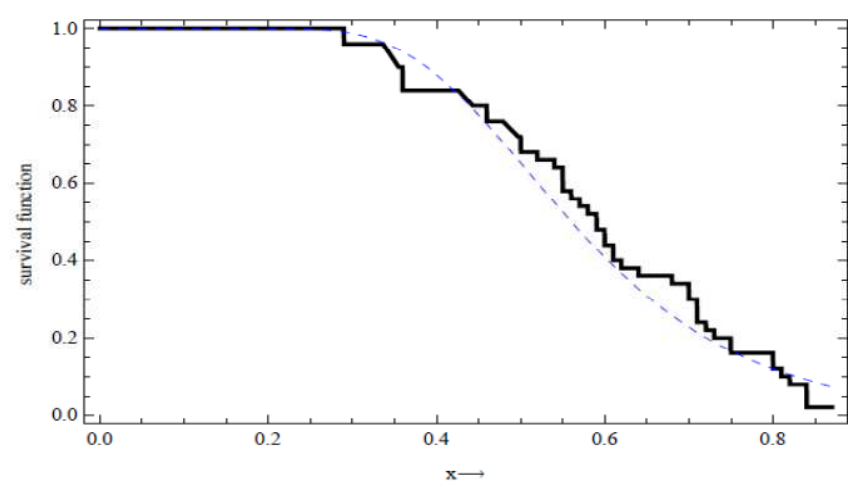

Fig 1. Empirical (bold line) and the estimated. (dotted lines) survival

\begin{tabular}{|c|c|c|c|c|c|c|c|c|}
\hline$i$ & 1 & 2 & 3 & 4 & 5 & 6 & 7 & 8 \\
\hline$x_{i, m, n}$ & 0.29 & 0.35 & 0.44 & 0.44 & 0.46 & 0.49 & 0.49 & 0.50 \\
\hline$y_{i, m, n}$ & 0.001 & 0.004 & 0.023 & 0.023 & 0.030 & 0.043 & 0.043 & 0.047 \\
\hline$i$ & 9 & 10 & 11 & 12 & 13 & 14 & 15 & \\
\hline$x_{i, m, n}$ & 0.52 & 0.55 & 0.55 & 0.59 & 0.61 & 0.64 & 0.80 & \\
\hline$y_{i, m, n}$ & 0.058 & 0.075 & 0.075 & 0.100 & 0.114 & 0.136 & 0.272 & \\
\hline
\end{tabular}
functions for [22].

Table 3. Original and transformed progressive first-failure samples for Wingo [21] data-set.

To illustrate the computation of the method proposed in previous sections, a progressive first-failure censoring scheme was conducted with $k=2, n=25, m=15$, and censoring scheme $R_{1}=10, R_{i}=0, i=2, \ldots m$, is generated from the original data. The observed and transformed data using the transformation $Y_{i: m: n}=-\ln \left(1-\exp \left(-X_{i: m: n}^{-\hat{\alpha}}\right)\right)$, are presented in 
Table 3, where $\hat{\alpha}=1.62$ is the estimate value of $\alpha$, which can be obtained by using the graphical method introduced by Balakrishnan and Kateri [22].

From (18), the MLE of the unknown parameter $\theta$ is 7.1537. Hence, using (19), the MLE of $C_{L}$ is 0.8999 . The lower lifetime limit $L$ is assumed to be 0.01399 , i.e. if the lifetime (in hours) of relief for arthritic patients exceeds 0.4078 , then a relief time for arthritic patients is defined as a conforming product. To deal with the product purchasers' concerns regarding operational performance, the conforming rate $P_{r}$ of products is required to exceed $80 \%$. Referring to Table 1 , the $C_{L}$ value is required to exceed 0.80 . Thus, the performance index value is set at $c=0.80$. The testing hypothesis:

$H_{0}: C_{L} \leq 0.80$ vs. $H_{1}: C_{L}>0.80$ is constructed. From $(23)$ the level $100(1-\gamma) \%$ lower confidence bound for $C_{L}$ is $L B_{M L}=0.854$. We observed the performance index value $c=0.80 \notin[0.854, \infty)$, hence the null hypothesis $H_{0}: C_{L} \leq 0.80$. is rejected. Thus one can conclude that the lifetime of relief for arthritic patients does meet the required level.

By using gamma prior given by (20) with hyperparameters are $a=2, b=1$, using (25) the Bayes estimate of $C_{L}$ is 0.9232 . From (30), with significance level $\gamma=0.05$, the $95 \%$ lower confidence bound for $C_{L}$ is

$$
L B_{B}=1-(1-0.9232)\left(\frac{\operatorname{CHIINV}(1-0.05,2(15+2))}{2(15+2)}\right)=0.8902 .
$$

Because of the performance index value $c=0.80 \notin[0.8902, \infty)$, we reject the null hypothesis $H_{0}: C_{L} \leq 0.80$. Hence, we can also conclude that the lifetime of relief for arthritic patients does meet the required level for the Bayesian approach.

Example 2. (Simulated Data): For given $\theta=1.5$ and $\alpha=1.62$, a progressive first-failure-censored sample was generated from EF distribution (1). By taken $n=25, m=15, k=2, \quad R_{i}=0, i=1,2, \ldots m, \quad i \neq 8, \quad$ and $R_{8}=10$. And by using the following transformation:

$$
Y_{i: m: n}=-\ln \left(1-\exp \left(-X_{i: m: n}^{-1.62}\right)\right), i=1,2, \ldots m .
$$

The observed and the transformed data are listed in Table 4. Here; the lower lifetime limit $L$ is assumed to be 0.06667 . The conforming rate $P_{r}$ of operational performances is required to exceed $80 \%$. Referring to Table 1 , the $C_{L}$ value will exceed 0.80 . Thus, the performance index value is set at $c=0.80$. Based on the generated progressive first failure censored sample $y_{i}, i=1,2, \ldots m$, using (18) the MLE of the unknown parameter $\theta$ is computed to be 1.5356 and from (19) $\hat{C}_{L_{M L}}=0.8976$. Then the 95\% lower confidence bound ( $L B_{M L}$ ) for $C_{L}$ is 0.8506 . Because of the performance index value $c=0.80 \notin[0.8506, \infty)$, the null hypothesis $H_{0}: C_{L} \leq 0.80$ is rejected. Thus one can conclude that the lifetime performance index of the product does meet the required level.

For Bayesian computation it is assumed that the prior distribution of $\theta$ has gamma distribution given by Eq. (20) with $a=2$ and $b=2$. With $L=0.06667$, using (25) the Bayes estimate of $C_{L}\left(\hat{C}_{L B}\right)$ is 0.9037 . From (30), with significance level $\gamma=0.05$, the $95 \%$ lower confidence bound $\left(L B_{B}\right)$ for $C_{L}$ is 0.8623 . Also, since $c=0.80 \notin[0.8623, \infty)$, the null hypothesis $H_{0}: C_{L y} \leq 0.80$ is rejected. Thus, we can conclude that the lifetime performance index of the product meets the required level.

\subsection{The Monte Carlo Simulation Study}

In this subsection, we report the obtained results of a simulation study, which was carried out by software Mathematica Version 8, to compare the performances of the MLEs and Bayes estimates based on the progressive first-failure censored schemes. This simulation has done by different values of $n$ (number of groups), different effective sample sizes $m$, different number of items in each group $k$, and by choosing $\alpha=0.9864$ and $\theta=1.4985$ in all the cases. We have used four progressive censoring schemes as:

Scheme 1: $R_{1}=n-m, R_{i}=0$ for $i \neq 1$.

Scheme 2: $R_{m}=n-m, R_{i}=0$ for $i \neq m$.

And

Scheme 3: $\quad R_{\frac{m+1}{2}}=n-m, R_{i}=0$ for $i \neq \frac{m+1}{2}$.

To generate progressively first-failure censored samples from Exponentiated Frechet (EF) distribution, we used the algorithm proposed by Balakrishnan and Aggarwala [5], with the fact that, the progressive first-failure censored sample $x_{1: m: n: k}^{R}, x_{2: m: n: k}^{R}, \ldots, x_{m: m: n: k}^{R}$ with distribution function $F(x)$, can be viewed as a progressive type II censored sample from a population with distribution function $1-(1-F(x))^{k}$. For each case the ML and Bayes estimates of lifetime performance index $C_{L}$ are computed based on 1000 replications. We evaluated Bayesian estimates under the assumption that hyperparameters take values as: $(a, b)=$ $(1,1),(2,2)$ and $(4,4)$. With $L=0.01$, the average means (first entry), mean squared errors (MSEs) (second entry) and the coverage probabilities (third entry) of $C_{L}$ for some selected choices of $n, m$ and $k$, are reported in Tables 5 and 6. It is observed that as $n$ (sample size) and $m$ (effective sample size) increases the performances become better in terms of MSEs in all cases considered. From Tables 5 and 6 we can see that the Bayes estimates are better than their corresponding maximum likelihood estimates for the considered cases. Moreover, it is observed that the coverage probabilities of one-sided credible interval and one-sided confidence interval for lifetime performance index $C_{L}$ close to the desired level of 0.95 . Also the censoring scheme $R=(n-m, 0, \ldots, 0)$ is most efficient for all choices; it usually provides the smallest MSE for all estimators. 
Table 4. The generated progressive first-failure censored sample for Example 2.

\begin{tabular}{|c|c|c|c|c|c|c|c|c|}
\hline$i$ & 1 & 2 & 3 & 4 & 5 & 6 & 7 & 8 \\
\hline$x_{i, m, n}$ & 0.4059 & 0.4725 & 0.5354 & 0.5731 & 0.6215 & 0.6453 & 0.6574 & 0.6636 \\
\hline$y_{i, m, n}$ & 0.0135 & 0.0350 & 0.0660 & 0.0889 & 0.1224 & 0.1403 & 0.1497 & 0.1546 \\
\hline$i$ & 9 & 10 & 11 & 12 & 13 & 14 & 15 & \\
\hline$x_{i, m, n}$ & 0.6749 & 0.7603 & 0.7636 & 0.7853 & 0.9151 & 0.9875 & 1.4276 & \\
\hline$y_{i, m, n}$ & 0.1636 & 0.2362 & 0.2392 & 0.2585 & 0.3786 & 0.4468 & 0.8445 & \\
\hline
\end{tabular}

Table 5. Average mean, the corresponding MSEs and coverage probabilities of $C_{L}$, with $k=2$.

\begin{tabular}{|c|c|c|c|c|c|c|c|c|}
\hline n & $\mathbf{m}$ & Scheme & MLE & Bayes & MLE & Bayes & MLE & Bayes \\
\hline & & & & $\mathrm{a}=\mathrm{b}=2$ & & $\mathrm{a}=\mathrm{b}=1$ & & $\mathrm{a}=\mathrm{b}=4$ \\
\hline \multirow[t]{9}{*}{25} & 15 & $\left(10,14^{0}\right)$ & 0.9839 & 0.9851 & 0.9839 & 0.9846 & 0.9838 & 0.9859 \\
\hline & & & 0.1867 & 0.0975 & 0.2124 & 0.1448 & 0.2065 & 0.0735 \\
\hline & & & (0.949) & $(0.935)$ & $(0.954)$ & $(0.945)$ & $(0.957)$ & $(0.922)$ \\
\hline & & $\left(7^{0}, 10,7^{0}\right)$ & 0.9841 & 0.9853 & 0.9839 & 0.9846 & 0.9837 & 0.9858 \\
\hline & & & 0.2011 & 0.1064 & 0.2250 & 0.1497 & 0.2264 & 0.0771 \\
\hline & & & $(0.952)$ & $(0.938)$ & $(0.946)$ & $(0.942)$ & $(0.946)$ & $(0.926)$ \\
\hline & & $\left(14^{0}, 10\right)$ & 0.9838 & 0.9851 & 0.9839 & 0.9846 & 0.9836 & 0.9858 \\
\hline & & & 0.2190 & 0.1090 & 0.2210 & 0.1490 & 0.2296 & 0.0774 \\
\hline & & & $(0.955)$ & $(0.943)$ & $(0.953)$ & $(0.951)$ & $(0.947)$ & $(0.922)$ \\
\hline \multirow[t]{9}{*}{35} & 25 & $\left(10,24^{0}\right)$ & 0.9844 & 0.9851 & 0.9844 & 0.9848 & 0.9845 & 0.9857 \\
\hline & & & 0.0952 & 0.0653 & 0.1068 & 0.0867 & 0.1075 & 0.0599 \\
\hline & & & $(0.962)$ & $(0.947)$ & $(0.957)$ & $(0.951)$ & $(0.943)$ & $(0.912)$ \\
\hline & & $\left(12^{0}, 10,12^{0}\right)$ & 0.9843 & 0.9850 & 0.9842 & 0.9846 & 0.9845 & 0.9857 \\
\hline & & & 0.1257 & 0.0838 & 0.1146 & 0.0913 & 0.1110 & 0.0606 \\
\hline & & & $(0.938)$ & $(0.926)$ & $(0.966)$ & $(0.963)$ & $(0.95)$ & $(0.918)$ \\
\hline & & $\left(24^{0}, 10\right)$ & 0.9844 & 0.9851 & 0.9844 & 0.9848 & 0.9841 & 0.9854 \\
\hline & & & 0.1053 & 0.0720 & 0.1083 & 0.0877 & 0.1291 & 0.0637 \\
\hline & & & $(0.946)$ & $(0.935)$ & $(0.953)$ & $(0.948)$ & $(0.959)$ & $(0.924)$ \\
\hline \multirow[t]{9}{*}{45} & 35 & $\left(10,34^{0}\right)$ & 0.9847 & 0.9851 & 0.9847 & 0.9850 & 0.9846 & 0.9855 \\
\hline & & & 0.0635 & 0.0491 & 0.0692 & 0.0605 & 0.0737 & 0.0475 \\
\hline & & & $(0.96)$ & $(0.951)$ & $(0.943)$ & $(0.934)$ & $(0.956)$ & $(0.926)$ \\
\hline & & $\left(17^{0}, 10,17^{0}\right)$ & 0.9846 & 0.9851 & 0.9845 & 0.9847 & 0.9846 & 0.9854 \\
\hline & & & 0.0701 & 0.0540 & 0.0743 & 0.0637 & 0.0754 & 0.0478 \\
\hline & & & $(0.951)$ & $(0.935)$ & $(0.957)$ & $(0.952)$ & $(0.953)$ & $(0.926)$ \\
\hline & & $\left(34^{0}, 10\right)$ & 0.9846 & 0.9851 & 0.9845 & 0.9847 & 0.9845 & 0.9854 \\
\hline & & & 0.0721 & 0.0555 & 0.0779 & 0.0668 & 0.0755 & 0.0471 \\
\hline & & & $(0.951)$ & $(0.933)$ & $(0.953)$ & $(0.945)$ & $(0.955)$ & $(0.920)$ \\
\hline
\end{tabular}

Note: Corresponding to each scheme, the first figure represents the average estimates, with the corresponding MSEs reported below $\rceil$ it in parentheses and multiply by $10^{-4}$ 
Table 6. Average mean, the corresponding MSEs and coverage probabilities of $C_{L}$, with $k=4$

\begin{tabular}{|c|c|c|c|c|c|c|c|c|}
\hline n & $\mathbf{m}$ & Scheme & MLE & Bayes & MLE & Bayes & MLE & Bayes \\
\hline & & & & $a=b=2$ & & $\mathrm{a}=\mathrm{b}=1$ & & $\mathrm{a}=\mathrm{b}=4$ \\
\hline \multirow[t]{9}{*}{25} & 15 & $\left(10,14^{0}\right)$ & 0.9838 & 0.9851 & 0.9838 & 0.9845 & 0.9839 & 0.9859 \\
\hline & & & 0.2100 & 0.1070 & 0.2000 & 0.1390 & 0.1978 & 0.0732 \\
\hline & & & $(0.951)$ & $(0.941)$ & $(0.955)$ & $(0.949)$ & $(0.953)$ & $(0.929)$ \\
\hline & & $\left(7^{0}, 10,7^{0}\right)$ & 0.9837 & 0.9850 & 0.9838 & 0.9846 & 0.9838 & 0.9859 \\
\hline & & & 0.2260 & 0.1100 & 0.2380 & 0.1600 & 0.2200 & 0.0789 \\
\hline & & & $(0.956)$ & (0.949) & $(0.949)$ & $(0.941)$ & $(0.947)$ & $(0.922)$ \\
\hline & & $\left(14^{0}, 10\right)$ & 0.9835 & 0.9849 & 0.9838 & 0.9845 & 0.9841 & 0.986 \\
\hline & & & 0.2400 & 0.1180 & 0.2200 & 0.1500 & 0.2040 & 0.0781 \\
\hline & & & $(0.956)$ & $(0.943)$ & $(0.956)$ & $(0.947)$ & $(0.947)$ & $(0.921)$ \\
\hline \multirow[t]{9}{*}{35} & 25 & $\left(10,24^{0}\right)$ & 0.9842 & 0.9849 & 0.9845 & 0.9848 & 0.9843 & 0.9855 \\
\hline & & & 0.1100 & 0.0732 & 0.0957 & 0.0781 & 0.1080 & 0.0574 \\
\hline & & & $(0.956)$ & $(0.948)$ & $(0.956)$ & $(0.950)$ & $(0.960)$ & $(0.928)$ \\
\hline & & $\left(12^{0}, 10,12^{0}\right)$ & 0.9842 & 0.985 & 0.9844 & 0.9848 & 0.9843 & 0.9855 \\
\hline & & & 0.1140 & 0.0753 & 0.1066 & 0.0861 & 0.1160 & 0.0610 \\
\hline & & & $(0.962)$ & $(0.952)$ & $(0.955)$ & $(0.949)$ & $(0.945)$ & $(0.928)$ \\
\hline & & $\left(24^{0}, 10\right)$ & 0.9844 & 0.9851 & 0.9843 & 0.9847 & 0.9844 & 0.9856 \\
\hline & & & 0.1200 & 0.0820 & 0.1100 & 0.0900 & 0.1158 & 0.0620 \\
\hline & & & $(0.943)$ & $(0.932)$ & $(0.949)$ & $(0.947)$ & $(0.950)$ & $(0.923)$ \\
\hline \multirow[t]{9}{*}{45} & 35 & $\left(10,34^{0}\right)$ & 0.9846 & 0.985 & 0.9845 & 0.9848 & 0.9846 & 0.9855 \\
\hline & & & 0.0707 & 0.0540 & 0.0760 & 0.0655 & 0.0724 & 0.0466 \\
\hline & & & $(0.941)$ & $(0.928)$ & $(0.948)$ & $(0.945)$ & $(0.945)$ & $(0.922)$ \\
\hline & & $\left(17^{0}, 10,17^{0}\right)$ & 0.9846 & 0.9851 & 0.9846 & 0.9848 & 0.9845 & 0.9854 \\
\hline & & & 0.0711 & 0.0544 & 0.076 & 0.0658 & 0.0788 & 0.0497 \\
\hline & & & $(0.947)$ & $(0.933)$ & (0.939) & $(0.930)$ & $(0.948)$ & $(0.922)$ \\
\hline & & $\left(34^{0}, 10\right)$ & 0.9845 & 0.9850 & 0.9845 & 0.9847 & 0.9846 & 0.9855 \\
\hline & & & 0.0730 & 0.0557 & 0.0800 & 0.0690 & 0.0734 & 0.0475 \\
\hline & & & $(0.946)$ & $(0.935)$ & $(0.949)$ & $(0.945)$ & $(0.947)$ & $(0.925)$ \\
\hline
\end{tabular}

Note: Corresponding to each scheme, the first figure represents the average estimates, with the corresponding MSEs reported below it in parentheses and multiply by $10^{-4}$

\section{Conclusions}

In this paper, adopting Bayesian and non-Bayesian approaches, various estimators for $C_{L}$ were derived based on progressive first-failure censoring samples arising from the EF distribution. A ML procedure at level $\gamma$ and a Bayesian rule for testing the null hypothesis against the alternative $H_{1}: C_{L}>c$ were provided. Also, various $100(1-\gamma) \%$ confidence and credible intervals for $C_{L}$ using Bayesian and non-Bayesian approaches were constructed.

\section{References}

[1] M. Frechet, Sur la loi de probabilit de l'ecart maximum, Ann. Soc. Polon. Math. (Cracovie), 6, (1927), 93-116.
[2] S. Kotz, and S. Nadarajah, Extreme Value Distribution: Theory and Applications. Imperial College Press, London, (2000).

[3] S. Nadarajah, and S. Kotz, The Exponentiated Type Distributions Acta Appl. Math., 92(2), (2006), 97--111.

[4] N. Balakrishnan, and R. Aggarwala, Progressive Censoring: Theory, Methods and Applications. Boston: Birkhauser Publishers, (2000).

[5] N. Balakrishnan, Progressive censoring methodology: An appraisal. Test 16, (2007), 211--296.

[6] L.G. Johnson, Theory and Technique of Variation Research, Elsevier, Amsterdam, (1964).

[7] S.-J. Wu and C. Kus, On estimation based on progressive first-failure censored sampling, Comput. Statist. Data Anal. 53(10), (2009), pp. 3659--3670.

[8] S. J. Wu and S. R. Huang, Progressively first-failure censored reliability sampling plans with cost constraint, Computational Statistics and Data Analysis, 56, (2012), 2018-2030. 
[9] A.A. Soliman, A.H. Abd Ellah, N.A. Abou-Elheggag, and A.A. Modhesh, Estimation from Burr type XII distribution using progressive first failure censored data, Journal of Statistical Computation and Simulation, 83,( 2013), 2270-2290

[10] D.C Montgomery, Introduction to Statistical Quality Control, John Wiley and SonsInc., New York, (1985).

[11] V.E Kane, Process capability indices, Journal of Quality Technology 18, (1986), 4152.

[12] L. I. Tong, K. S. Chen, and H. T Chen, Statistical testing for assessing the performance of lifetime index of electronic components with exponential distribution, Journal of Quality Reliability Management, vol. 19, (2002), pp. 812--824, .

[13] J.W. Wu, H.M. Lee, and C.L. Lei, Computational testing algorithmic procedure of assessment for lifetime performance index of products with two-parameter exponential distribution, Applied Mathematics and Computation 190, (2007), 116.125

[14] W.C. Lee, J.W. Wu, and C.W. Hong, Assessing the lifetime performance index of products with the exponential distribution under progressively type-II right censored samples, Journal of Computational and Applied Mathematics 231, (2009), 648--656.

[15] M.V. Ahmadi, M. Doostparast and J. Ahmadi, Estimating the lifetime performance index with Weibull distribution based on progressive first-failure censoring scheme, Journal of Computational and Applied Mathematics 239, (2013), 93--102.
[16] W.C. Lee, J.W. Wu, C.W. Hong, and S.F. Hong, Evaluating the Lifetime Performance Index Based on the Bayesian Estimation for the Rayleigh Lifetime Products with the Upper Record Values, Journal of Applied Mathematics, Volume, (2013), Article ID 547209, 13 pages, http://dx.doi.org/10.1155/2013/547209.

[17] M.V. Ahmadi, M. Doostparast and J. Ahmadi, Statistical inference for the lifetime performance index based on generalised order statistics from exponential distribution, International Journal of Systems Science, http://dx.doi.org/10.1080/00207721.2013.809611

[18] M. Ahsanullah Generalized Order Statistics From Exponential Distribution, Journal of Statistical Planning and Inference, 85, (2000), 85--91.

[19] G. Casella, and R.L. Berger, Statistical Inference, second ed., Duxbury, Pacific Grove, CA, (2002).

[20] D.R. Wingo, Maximum likelihood methods for fitting the Burr type XII distribution to life test data, Biome. J. 25, (1983), pp. $77--84$.

[21] S.-F. Wu, C.-C. Wu, Y.-L. Chen, Y.-R. Yu, and Y.P. Lin, Interval estimation of a two-parameter Burr-XII distribution under progressive censoring, Statistics 44(1), (2010), pp. 77--88.

[22] N. Balakrishnan, and M. Kateri, On the maximum likelihood estimation of parameters of Weibull distribution based on complete and censored data, Statist. Probab. Lett. 78, (2008), pp. 2971--2975. 